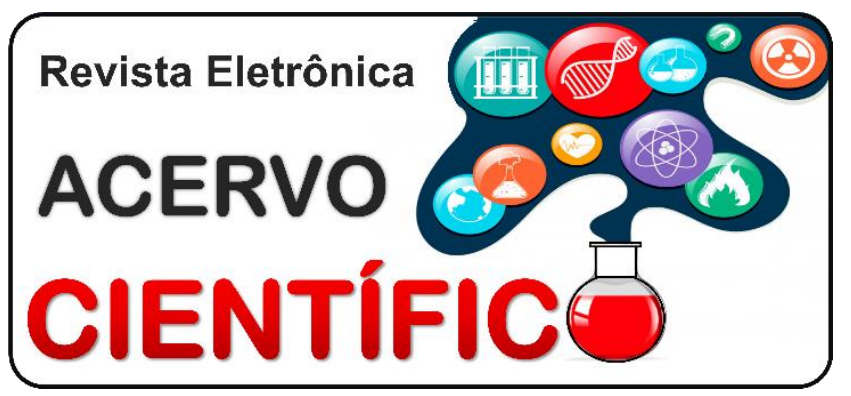

REVISÃO BIBLIOGRÁFICA

Recebido em: 2/2020

Aceito em: 2/2020

Publicado em: 5/2020

\title{
Prevalência dos estudos que avaliam a associação entre síndrome do pé diabético e suas complicações: uma análise a partir do instrumento Strobe
}

\author{
Prevalence studies evaluating the association between diabetic foot syndrome and its \\ complications: an analysis from the the Strobe instrument
}
Prevalencia de estúdios que evaluan la asociación entre el síndrome del pie diabético y sus complicaciones: um análisis del instrumento de Strobe

Ana Paula Ramos de Souza1*, Ana Carolina Fonseca Tavares ${ }^{1}$, Murilo Brandão Pimenta ${ }^{1}$, Ana Carolina Damasceno Corrêa1, Deusa Meriam da Silva Brito ${ }^{1}$, Brenda Nazaré Gomes Andriolo'.

Resumo: Avaliar a qualidade dos estudos observacionais transversais por meio da aplicação da iniciativa STROBE (Strengthening the Reporting of Observational Studies in Epidemiology). Estudo analítico, do tipo transversal e retrospectivo a partir de estudos recrutados na base de dados PUBMED com os seguintes descritores de busca: ("Diabetic Foot"[Mesh]) AND ("Prevalence"[Mesh] OR "Cross-Sectional Studies"[Mesh] ) os termos meSH para diabetic foot incluíam os seguintes termos: Foot, Diabetic,Diabetic Feet, Feet, Diabetic foot Ulcer, Diabetic. O estudo foi conduzido na Faculdade Metropolitana da Amazônia, em Belém do Pará. Dentro dos artigos analisados, 10,5\% continham um domínio "Discussão" inadequado, $31,98 \%$ continham o domínio "Resultado" inadequado. O tópico "Outras Informações" foi avaliado inadequado $21,05 \%$ das vezes; "Introdução" inadequado 10,53\% das vezes, domínio "Título e Resumo" inadequado 5,26\% das vezes. Podese concluir de antemão que a iniciativa STROBE mostra-se como importante ferramenta para guiar autores que desejam realizar pesquisas, sobretudo transversais, corroborando para uma melhor estruturação metodológica e qualidade superior dos estudos.

Palavras-chave: Pé diabético, Prevalência, Complicações do diabetes.

\begin{abstract}
Assess the quality of cross-sectional studies through the application of the STROBE initiative (Strengthening the Reporting of Observational Studies in Epidemiology). Analytical study of cross-sectional and retrospective from studies recruited in the database PUBMED with the following search keywords: ("Diabetic Foot" [Mesh]) AND ("Prevalence" [Mesh] OR "Cross-Sectional Studies" [ Mesh]) MeSH terms for diabetic foot included the following terms: Foot, diabetic, diabetic Feet, Feet, diabetic foot Ulcer, diabetic. The study was conducted in the Amazon Metropolitan College in Belém do Pará. Among the articles analyzed, $10.5 \%$ had a domain "talk" inappropriate, $31.98 \%$ contained the area "Result" inappropriate. The topic "Further Information" was rated inadequate $21.05 \%$ of the time; "Introduction" inappropriate $10.53 \%$ of the time domain "title and summary" inappropriate $5.26 \%$ of the time. It can be concluded beforehand that the STROBE Initiative is shown as an important tool to guide authors who wish to conduct research, particularly cross, corroborating a better methodological structure and superior quality of the studies. This further studies are needed to examine the quality used in cross-sectional studies by STROBE tool.
\end{abstract}

Keywords: Diabetic foot, Prevalence, Diabetes complications.

\footnotetext{
${ }^{1}$ Centro Universitário Metropolitano da Amazônia (UNIFAMAZ), Belém - Pará.

*E-mail: anapaula.ramoss@hotmail.com
} 
Resumen: Evaluar la calidad de los estudios observacionales transversales a través de la aplicación de la iniciativa STROBE (Fortalecimiento de la notificación de estudios observacionales en epidemiología). Estudio analítico, transversal y retrospectivo basado en estudios reclutados de la base de datos PUBMED con los siguientes descriptores de búsqueda: ("Pie diabético" [Malla]) Y ("Prevalencia" [Malla] O "Estudios transversales" [ Malla]) los términos meSH para pie diabético incluyeron los siguientes términos: pie, diabético, pies diabéticos, pies, úlcera del pie diabético, diabético. El estudio se realizó en la Faculdade Metropolitana da Amazônia, en Belém do Pará. Dentro de los artículos analizados, el 10.5\% contenía un dominio inapropiado de "Discusión", el 31.98\% contenía un dominio inapropiado de "Resultado". El tema "Otra información" fue calificado como inapropiado el $21.05 \%$ del tiempo; Inadecuada "Introducción" $10.53 \%$ del tiempo, dominio "Título y Resumen" inadecuado $5.26 \%$ del tiempo. Se puede concluir de antemano que la iniciativa STROBE demuestra ser una herramienta importante para guiar a los autores que desean llevar a cabo investigaciones, sobre todo transversales, que corroboren una mejor estructuración metodológica y una calidad superior de los estudios.

Palabras clave: Pie diabético, Prevalencia, Complicaciones de la siabetes.

\section{INTRODUÇÃO}

A diabetes é uma enfermidade grave e frequente na população adulta haja vista atingir até $7 \%$ da população brasileira e o país ser $4^{\circ}$ país com mais casos da doença no mundo, definida pela diminuição da sensibilidade da Insulina humana à glicose que o corpo ingere até perda total da produção desse hormônio pelo pâncreas e está associada a um aumento da mortalidade por complicações macro e microvasculares tais como retinopatia diabética (problemas oculares), problemas renais interferindo na taxa de filtração deste órgão fazendo o corpo humano acumular substâncias que deveriam ser eliminadas e o pé diabético, embora sejam ainda escassos estudos relativos às complicações (CAMPANA AO, et al., 2001; BRASIL, 2002; ALTMAN DG e MOHER D, 2005; FREESE E, 2006).

O pé diabético, segundo a definição do Consenso Internacional sobre Pé Diabético (BRASIL, 2001), é uma infecção, ulceração e/ou destruição dos tecidos profundos associadas a anormalidades neurológicas e vários graus de doença vascular periférica nos membros inferiores. O paciente quando perde a sensibilidade do membro inferior, não percebe micro/macro lesões no pé (principalmente na parte inferior) associado a fragilidade da cicatrização faz desenvolver uma úlcera crônica. Essa síndrome representa uma das mais importantes complicações crônicas da diabetes, sendo a causa mais comum de amputações não traumáticas de membros inferiores em países industrializados, ocorrendo em $15 \%$ dos diabéticos e é responsável por $6 \%$ a $20 \%$ das hospitalizações (BRASIL, 2001; BRASIL 2001; CARVALHO CBM, et al., 2004).

Dessa forma, o diagnóstico desta situação pode e deve ser feito precocemente, já que uma adequada vigilância e assistência à saúde, aliadas a mudanças no estilo de vida da população e o controle da glicemia podem retardar o aparecimento da própria doença (diabetes) e prevenir suas complicações tais como acima citadas (BRASIL, 2002; FREESE E, 2006).

Os problemas do pé diabético, são uma complicação comum da diabetes mellitus, com uma prevalência de $23-42 \%$ para a neuropatia diabética, 9-23\% - para a doenças vasculares e 5-7\% - para a ulceração do pé que provoca incômodo, possibilidade de infecção até amputação do membro. Estes, resultam em importantes consequências médicas, sociais e económicas para os doentes, suas famílias e a sociedade (FREESE E, 2006). Para tanto, o desenvolvimento de pesquisas que atendam o protocolo STROBE faz com que os leitores/público destinado das pesquisas entendam com mais facilidade e rapidez suas informações, diminuindo o risco de dúvidas nas informações.

Na última década, ocorreu um nítido crescimento do número de artigos científicos que são diretamente relevantes para a prática da Medicina haja vista o profissional médico utilizar estes estudos para conduzir seu manejo, exame físico, discussão de casos e todos os aspectos que envolvem a Medicina. No entanto, junto a esse rápido crescimento, tem-se dado pouca atenção à transparência de como são descritos domínios desses estudos, dificultando a avaliação de seus pontos fracos e fortes e, em consequência, a generalização de seus resultados e isto pode gerar consequências para a prática médica pois a maioria dos médicos não 
conseguem diferenciar um estudo bom de outro ruim pela estatística ou metodologia, possibilitando desenvolver estudos sem relevância médica (COSTA L, 2011).

Mais recentemente, grupos de pesquisadores ao redor do mundo têm feito tentativas bem-sucedidas, para resolver esse problema por meio da criação de diretrizes que auxiliam os pesquisadores não apenas na preparação dos manuscritos, mas também garantem que detalhes importantes relacionados ao delineamento do estudo sejam devidamente descritos, como exemplo a iniciativa Strengthening the Reporting of Observational Studies in Epidemiology (STROBE), a qual desenvolveu uma lista com 22 itens de recomendação sobre o que deveria ser incluído em estudos observacionais transversais (Declaração STROBE), melhorando a metodologia dos estudos e por conseguinte os resultados que os médicos necessitam ao analisar o estudo (HADDAD N, 2004; SHEIKHA et al., 2007).

O STROBE é composto por informações que devem estar presentes nos componentes título, resumo, introdução, metodologia, resultados e discussão de artigos que descrevem estudos observacionais científicos. Dezoito itens (18) são de estudo de coorte, seccionais e caso-controle, e quatro (4) são específicos para cada um desses três tipos de estudo diminuindo o risco de mal-entendido na leitura ou dificuldade de compreensão (HADDAD N, 2004).

A Iniciativa STROBE foi originária de uma pesquisa publicada na língua inglesa. Após isso, grupos independentes de pesquisadores de vários países traduziram a listagem e os princípios da Iniciativa STROBE, com o objetivo de disseminar e espalhar para o público cada vez maior os princípios que deveriam nortear e direcionar a descrição de estudos observacionais (HADDAS N, 2004).

Os desenhos de estudo transversais são estudos em que a exposição a causa está passível de presente ao efeito no mesmo momento ou intervalo de tempo analisado (HADDAD N, 2004). O estudo transversal pode ser usado como um estudo analítico, ou seja, para avaliar hipóteses de associações entre exposição ou características e evento (VON ELM E, et al., 2007).

Esse modelo possui metáfora de semelhante como uma "fotografia" ou corte instantâneo que é feito em uma população por meio de uma amostra, examinando-se nos integrantes da casuística ou grupo populacional, a presença ou ausência da exposição de algo à este grupo e a presença ou ausência do efeito na mesma população (ou doença) (SHEIKH L, et al., 2007). Possui como principais benefícios o fato de necessitar de menos tempo pra ser desenvolvido quando comparado com os outros tipos de estudo e serem de baixo custo (FREESE E, 2006).

Foram selecionadas pesquisas transversais associadas às complicações de Diabetes Mellitus tipo I e II devido a importante demanda dessa doença nos serviços de saúde de Belém - Pará - Brasil, na qual os pesquisadores possuem contato com a comunidade (HADDAD N, 2004; SHEIKH L, et al., 2007; VON ELM E, et al., 2007).

O objetivo deste trabalho foi avaliar a qualidade dos estudos observacionais transversais já publicados sobre associação da Síndrome do Pé Diabético e suas complicações na literatura médica em uma base de dados por meio da aplicação da iniciativa STROBE. Além disso, este estudo buscou chamar a atenção para a concordância das recomendações internacionais ao escrever um estudo transversal com o auxílio da iniciativa STROBE.

\section{MÉTODOS}

O presente estudo é do tipo transversal, analítico e a sua amostra foi composta por todos os estudos recrutados na base de dados PUBMED, motor de busca de livre acesso à base de dados MEDLINE (Sistema Online de Busca e Análise de Literatura Médica) com os seguintes descritores de busca: ("Diabetic Foot"[Mesh]) AND ("Prevalence"[Mesh] OR "Cross-Sectional Studies"[Mesh] )

Os termos meSH para diabetic foot incluiam os seguintes termos: Foot, Diabetic,Diabetic Feet, Feet, Diabetic foot Ulcer, Diabetic. Foram incluídos artigos publicados em idioma inglês e idioma português, transversais, que avaliaram qualquer associação entre a prevalência de síndrome de pé diabético e suas 
complicações diversas. O estudo foi conduzido na Faculdade Metropolitana da Amazônia, Belém - Pará Brasil.

O procedimento metodológico utilizado para localizar os estudos foi o estabelecido a partir de um mecanismo de busca sensível que combinou termos oficiais e sinônimos de síndrome do pé diabético desde o início da indexação na base até 28 de outubro de 2016. Na sequência os autores principais selecionaram os estudos a partir da leitura dos títulos e resumos, de forma independente, que iriam compor a amostra.

A presente pesquisa analisou estudos publicados já previamente aprovados por Comitê de Ética em Pesquisa (CEP), por esta razão, não necessita de nova submissão, uma vez que nenhum indivíduo sofreu qualquer intervenção. Este projeto dependeu do aceite formal do orientador que foi assinado e foi conduzido buscando preservar os devidos aspectos éticos, mantendo a autenticidade das ideias, conceitos e definições dos autores pesquisados, bem como realizando as devidas citações e referências de acordo com as normas ABNT (Associação Brasileira de Normas Técnicas).

Os dados foram coletados utilizando uma planilha no software LibreOffice Calc. padronizada com as seguintes informações dos estudos: título e resumo, introdução, métodos, resultados, discussão e outras informações, cada tópico com suas subdivisões correspondentes, de acordo com os critérios da iniciativa STROBE. O presente estudo foi financiado com recursos próprios. Os artigos selecionados foram classificados pelos pesquisadores, com base nos critérios propostos pela iniciativa STROBE. Os itens que a compõem são relacionadas as informações que deveriam estar presentes nos capítulos referentes as sessões: título, resumo, introdução, metodologia, resultados e discussão de artigos científicos que descrevem estudos transversais (CARVALHO CBM, et al., 2011).

Para cada capítulo julgado, então, cada artigo foi classificado como: Adequado: se preencheu todos, senão a maioria, dos pré-requisitos da iniciativa STROBE para aquele capítulo, Inadequado: se não preencheu os pré-requisitos estabelecidos pela iniciativa STROBE para aquele capítulo. Os dados coletados foram tratados por métodos de estatística descritiva e as tabelas e gráficos foram elaborados, de acordo com as normas vigentes, no software LibreOffice Calc.

A proposta deste trabalho é realizar uma revisão das principais publicações acerca da associação entre DM e pé diabético. Como risco a probabilidade de que os autores deixem alguma informação ou publicação pertinente ao tema passe despercebida, bem como manipular dados ou alterar os dados, prejudicando a credibilidade desta pesquisa. Para prevenir tal risco os autores se comprometem em realizar um levantamento cuidadoso e sistemático de informações, a priori de forma independente e depois cruzando os artigos selecionados a fim de sensibilizar o refinamento da busca, reconhecendo e dando crédito à criação intelectual de outros autores ao expor fielmente de forma clara os dados coletados.

\section{RESULTADOS E DISCUSSÃO}

Um total de 366 artigos foram inicialmente recrutados na base de dados PUBMED em outubro de 2016, porém 341 não preencheram o critério de inclusão estabelecidos, seja por não se encaixarem no desenho do estudo (112), como coorte, caso controle, revisão sistemática, experimental e narrativa descritiva; por estarem em outra língua (98) como espanhol francês, polonês, alemão, holandês, húngaro, Eslováquia, chinês, lituânio, turco, japonês, hebreu, norueguês e italiano; ou por não associarem síndrome de pé diabético com as complicações mais prevalentes (141).

Finalmente, considerando os critérios de inclusão propostos pelo projeto, 25 artigos foram selecionados, sendo destes 19 disponíveis na íntegra e 6 não disponíveis na base de dados, exigindo requerimento perante o autor do mesmo ou pagamento de assinatura digital nas bibliotecas online (Figura 1).

Segundo a avaliação de qualidade dos estudos, de acordo com o preconizado pela iniciativa STROBE, são avaliados 6 tópicos referentes aos capítulos dos projetos (título/resumo, introdução, método, resultados, discussão, outras informações), totalizando 23 subtópicos que após a leitura de cada estudo foram classificados em adequado, inadequado e insuficiente (Tabela 1 e Tabela 2). 
Figura 1 - Fluxograma da seleção dos artigos para análise.

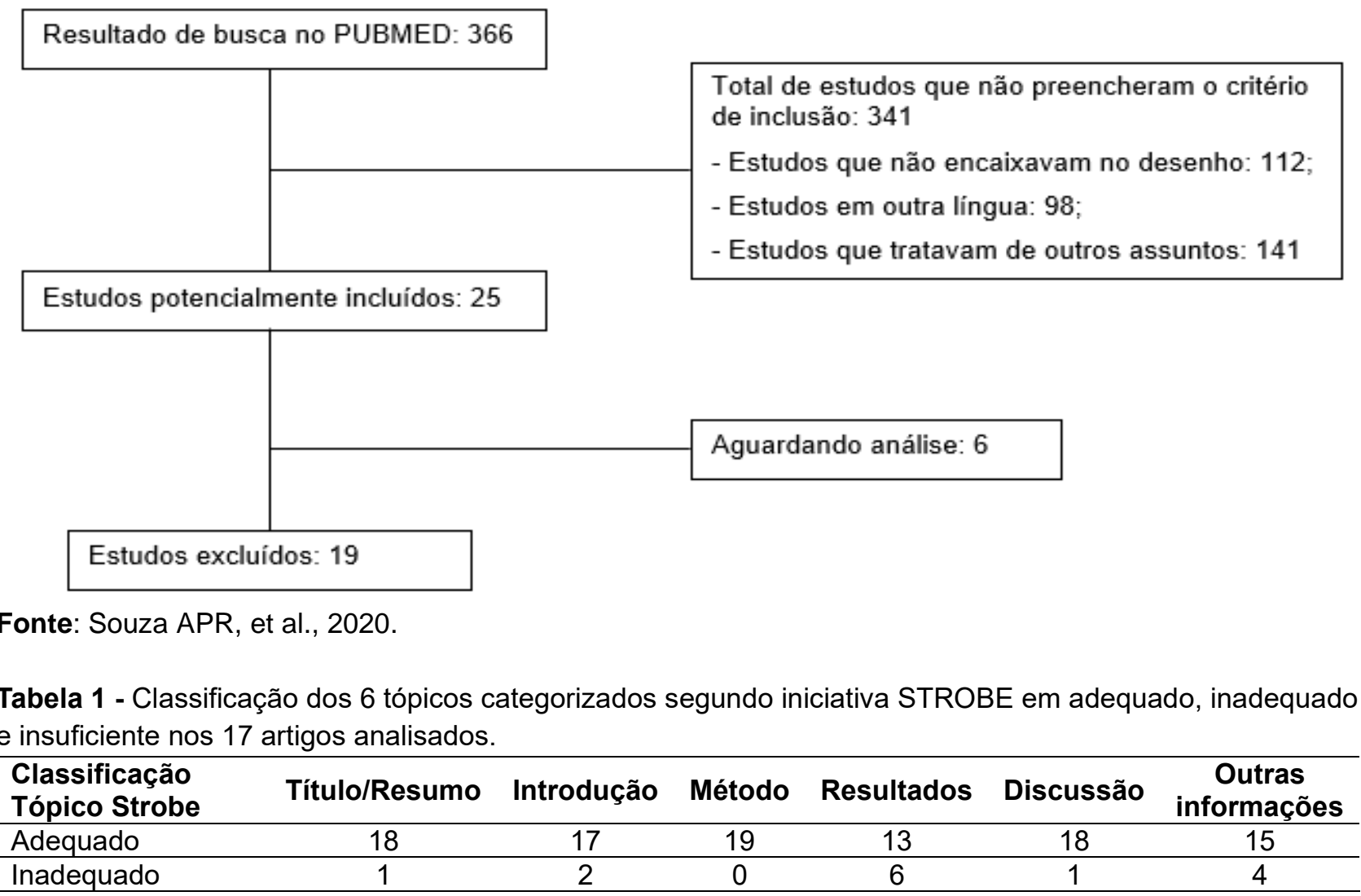

Fonte: Souza APR, et al., 2020.

Tabela 2 - Classificação dos 23 subtópicos categorizados segundo iniciativa STROBE em adequado, inadequado e insuficiente nos 17 artigos analisados.

\begin{tabular}{lll}
\hline Subtópicos Strobe/Classificação & Adequado & Inadequado \\
\hline 1a - Desenho do estudo no título e/ou resumo & $18(94,74 \%)$ & $1(5,26 \%)$ \\
1 b - Resumo informativo e equilibrado & $18(94,74 \%)$ & $1(5,26 \%)$ \\
2 - Contexto / Justificativa (Introdução) & $17(89,47 \%)$ & $2(10,53 \%)$ \\
3 - Objetivos (Introdução) & $18(94,74 \%)$ & $1(5,26 \%)$ \\
4 - Desenho do estudo (Método) & $19(100 \%)$ & 0 \\
5 - Contexto (Método) & $18(94,74 \%)$ & $1(5,26 \%)$ \\
- Participantes (Método) & $6(100 \%)$ & 0 \\
7 - Variáveis (Método) & $12(63,16 \%)$ & $7(36,84 \%)$ \\
8 - Fontes de dados/mensuração (Método) & $14(73,68 \%)$ & $5(26,32 \%)$ \\
- Viés (Método) & $7(36,84 \%)$ & $12(63,16 \%)$ \\
10 - Tamanho do estudo (Método) & $14(73,68 \%)$ & $5(26,32 \%)$ \\
11 - Variáveis quantitativas (Método) & $19(100 \%)$ & 0 \\
12 - Métodos estatísticos (Método) & $15(78,95 \%)$ & $4(21,05 \%)$ \\
13 - Participantes (Resultados) & $11(57,89 \%)$ & $8(42,11 \%)$ \\
14 - Dados descritivos (Resultados) & $15(78,95 \%)$ & $4(21,05 \%)$ \\
15 - Desfecho (Resultados) & $14(73,68 \%)$ & $5(26,32 \%)$ \\
16 - Resultados principais (Resultados) & $12(63,16 \%)$ & $7(36,84 \%)$ \\
17 - Outras análises (Resultados) & $10(57,89 \%)$ & $9(47,37 \%)$ \\
18 - Resultados principais (Discussão) & $18(94,74 \%)$ & $1(5,26 \%)$ \\
$19-$ Limitações (Discussão) & $11(57,89 \%)$ & $8(42,11 \%)$ \\
20 - Interpretação (Discussão) & $16(84,21 \%)$ & $3(15,79 \%)$ \\
21 - Generalização (Discussão) & $12(63,16 \%)$ & $7(36,84 \%)$ \\
22 - Financiamento (Outras informações) & $15(78,95 \%)$ & $4(21,05 \%)$ \\
\hline
\end{tabular}

Fonte: Souza APR, et al., 2020. 
Os resultados demonstraram uma grande inadequação quanto ao subtópico 9 (viés) da iniciativa STROBE. Os artigos analisados não planejaram uma estratégia de prevenção de viés, um subtópico muito relevante do tópico da metodologia, o que demonstra uma necessidade de adequação dessas publicações ao modelo proposto pela iniciativa STROBE. Os estudos transversais estão frequentemente expostos a vieses de informação (ASSOCIAÇÃO INTERNACIONAL DE EPIDEMIOLOGIA, 1973; BRASIL, 2001), e é por esse motivo que a padronização sugerida tem de ser adotada por pesquisadores de modo geral.

Os tópicos, "Método" e "Resultados" figuraram entre os que mais contiveram inadequações, a metodologia de um estudo é de fundamental importância para sua realização, o rigor empregado em sua elaboração demonstra meios de se reproduzir um certo tipo de pesquisa, além de fornecer dados de imensa relevância estatística para qualidade do estudo. O capítulo de resultados deve mostrar de forma clara os achados de um determinado estudo, o que, de acordo com o que foi encontrado no presente estudo não se observou. De um modo geral, os estudos deixavam de abordar, principalmente, os subtópicos de descrição de outras análises realizadas (SHEIKH L, 2007) (tais quais: análises de subgrupo, interação e sensibilidade) e razões para a perda nas diferentes etapas e o número de participantes em cada etapa.

O domínio "Discussão", o tópico mais importante de um estudo científico, para o bom entendimento e relevância do assunto perante a comunidade científica, foi marcado pela inadequação em dois subtópicos muito significantes que foram os itens: validade externa (SITTA EB, et al., 2010) e limitações que servem para avaliar o quanto os resultados de um estudo podem ser aplicados a outros indivíduos, ou seja, sua aplicabilidade e generalização em estudos posteriores e demonstrar os possíveis pontos fracos da execução de estudos respectivamente.

O domínio "Outros fatores" chama a atenção para o hábito de não dar mérito a colaboradores do estudo que participam, financiando, tão quanto, apoiando a realização de produções científicas de alta relevância para a comunidade médica, haja vista que, o suporte financeiro é de crucial importância para a realização de muitos estudos que requerem o uso de equipamentos específicos e de alto custo. Os achados deste estudo, demonstram que por vezes os autores deixam de incluir o agradecimento de seus colaboradores, influenciando negativamente na opinião do investimento financeiro do âmbito científico.

É consenso que a melhor forma de ensinar o acadêmico a ler e interpretar um artigo científico corretamente, com análise crítica, é através da experiência de desenvolver projetos científicos (BOTELHO F, et al., 2008). Nesse contexto, a ferramenta STROBE é essencial na elaboração de artigos claros, concisos e padronizados que facilitem a sua interpretação, reprodutibilidade e que evitem o desvio sistemático de um estudo.

A iniciativa STROBE apesar de ser um instrumento útil na avaliação de estudos observacionais não é a prova de falhas, por ter sido desenvolvida por um consenso pode vir a necessitar de alterações. Ferramentas como o STROBE, pois pode facilitar a descrição do estudo realizado para pesquisadores em início de carreira, sendo que, esses checklists deveriam ser analisados na construção do desenho do projeto (ONWUEGBUZIE AJ, et al., 2007; FERGUSON E, et al., 2002; MAC MAHON B, et al., 2007; HAIR JR, et al., 2009; KOROLJUNBERG M, 2010). Sendo uma opção que pode ser utilizada e manuseada pelos pesquisadores, a mais para direcionar e melhorar o desenvolvimento de estudos epidemiológicos observacionais, além da possibilidade de ser utilizada como bibliografia e referência de apoio para discentes do ensino superior, com objetivo de contribuir para a formação de novos pesquisadores na graduação, mestrados e doutorados (FERGUSON E, et al., 2002).

Este trabalho apresentou como limitação a restrição quanto a língua, uma vez que foram incluídas publicações somente em inglês e português, o que se mostra como um dos grandes pontos de imprecisão do presente trabalho, por possibilitar o viés de descarte de artigos que seriam importantes para a formulação dele. Outra limitação igualmente importante, foi o fato de realizar a busca em apenas uma base de dados (PUBMED) e abranger largamente a associação entre síndrome do pé diabético e suas complicações tendo como resultado pesquisas que não necessariamente buscavam a mesma linha de raciocínio, impossibilitando, portanto, uma análise precisa dos dados. 


\section{CONSIDERAÇÕES FINAIS}

Pode-se concluir de antemão que a iniciativa STROBE (Strengthening the Reporting of Observational Studies in Epidemiology) mostra-se como importante ferramenta para guiar autores que desejam realizar pesquisas, sobretudo transversais, corroborando para uma melhor estruturação metodológica e qualidade superior dos estudos. Dessa forma são necessários mais estudos e pesquisas a serem desenvolvidas que analisem a qualidade empregada nos estudos transversais através da ferramenta STROBE.

\section{AGRADECIMENTOS E FINANCIAMENTO}

Os autores expressam seus sinceros agradecimentos ao grupo de Medicina Baseada em Evidências da Universidade Estadual do Pará (UEPA).

\section{REFERÊNCIAS}

1. ALTMAN DG, MOHER D. Developing guidelines for reporting healthcare research: scientifi c rationale and procedures. Med Clin (Barc). 2005;125(Supl 1):8-13.

2. ASSOCIAÇÃO INTERNACIONAL DE EPIDEMIOLOGIA. Guia de Métodos de Enseñanza. IEA/OPS/OMS, Publ. Cient.266, 1973, 246p.

3. BRASIL. Departamento de Ações Programáticas Estratégicas, Secretaria de Políticas de Saúde. Ministério da Saúde. Plano de reorganização da atenção à hipertensão arterial e ao diabetes mellitus. Brasília: Ministério da Saúde; 2002.

4. BRASIL. Grupo de Trabalho Internacional sobre Pé Diabético. Consenso Internacional sobre pé diabético. Brasília: Secretaria de Estado do Distrito Federal; 2001.

5. BRASIL. Departamento de Atenção Básica, Secretaria de Políticas de Saúde. Ministério da Saúde. Diabetes e a reorganização da atenção. Informe da Atenção Básica 2001; 2(6).

6. BOTELHO F, et al. Epidemiologia explicada - O valor de prova (p). Acta Urológica 2008; 25(3): 55-7

7. CAMPANA AO, et al. Investigação científica na área médica. 1st ed. São Paulo: Manole; 2001

8. CARVALHO CBM, et al. Pé diabético: análise bacteriológica de 141 casos. Arq Bras Endocrinol Metab 2004; 48:398405COSTA L. Rev Bras Fisioter, São Carlos, v. 15, n. 4, p. 267-71, jul./ago. 2011

9. FERGUSON E, et al. Factors associated with success in medical school: systematic review of the literature. BMJ. 2002;324(7343):952-7.

10. FREESE E. Epidemiologia, políticas e determinantes das doenças crônicas não transmissíveis no Brasil. Recife: Editora Universitária da UFPE; 2006.

11. HADDAD N. Metodologia de estudos em ciências da saúde. 1st ed. Sao Paulo: Roca; 2004.

12. HAIR JR., et al. Análise multivariada de dados. 6. ed. Porto Alegre: Bookman, 2009.

13. KORO-LJUNGBERG, M. Validity, responsibility, and aporia. Qualitative Inquiry, v. 16, n. 8, p. 603-610, 2010.

14. MAC MAHON B, et al. Is there a dark phase of this STROBE? Epidemiology. 2007;18(6):791.

15. ONWUEGBUZIE AJ, et al. Validity and qualitative research: an oxymoron? Quality \& Quantity: International Journal of Methodology, v. 41, n. 2, p. 233-249, 2007.

16. SITTA EB, et al. A contribuição de estudos transversais na área da linguagem com enfoque em afasia. Rev. CEFAC [Internet]. 2010 Dec [cited 2016 Oct 28]; 12 (6): 1059-1066.

17. SHEIKH L, et al. A review of the methodological features of systematic reviews in maternal medicine. BMC Med. 2007; $5: 10$.

18. VON ELM E, et al. Strengthening the Reporting of Observational Studies in Epidemiology (STROBE) statement: guidelines for reporting observational studies. BMJ. 2007;335(7624):806-8. 